\title{
Antraquinonas e naftoquinonas do caule de um espécime de reflorestamento de Tectona grandi (Verbenaceae)
}

\author{
Rafael Y.O. Moreira ${ }^{1}$, Mara S.P. Arruda ${ }^{1 *}$, Alberto C. Arruda ${ }^{1}$, Lourivaldo S. Santos ${ }^{1}$, Adolfo \\ H. Müller ${ }^{1}$, Giselle M.S.P. Guilhon ${ }^{1}$, Alberdan S. Santos ${ }^{1}$, Evaristo Terezo ${ }^{2}$ \\ ${ }^{1}$ Departamento de Química, Centro de Ciência Exatas e Naturais, Universidade Federal do Pará, Rua Augusto \\ Corrêa s/n, 66075-110, Belém, Pará, Brasil, \\ ${ }^{2}$ Renar Consultoria Ltda, Rua dos Mundurucus 1553 / 902, B. Campos, 66025-660, \\ Belém, Pará, Brasil
}

\begin{abstract}
RESUMO: O fracionamento do extrato hexânico do caule de um espécime de reflorestamento de Tectona grandis (Verbenaceae), através de procedimentos fitoquímicos clássicos, levou ao isolamento das naftoquinonas lapachol e desidro- $\alpha$-lapachona e das antraquinonas tectoquinona e obtusifolina. As estruturas das substâncias foram caracterizadas através da análise de métodos espectrométricos de RMN. Este é o primeiro estudo fitoquímico de um espécime de reflorestamento de Tectona grandis, no Brasil, sendo o objetivo principal deste trabalho a comprovação da presença de tectoquinona em espécimes cultivados.
\end{abstract}

Unitermos: Tectona grandis, Verbenaceae, espécime de reflorestamento, antraquinonas, naftoquinonas

\begin{abstract}
Anthraquinones and naphtoquinones from the bark of Tectona grandis (Verbenaceae) reforestation specimen". The hexane extract of the bark of Tectona grandis (Verbenaceae) afforded two anthraquinones and two naphtoquinones. Their caracterizations were obtained through NMR spectroscopic techniques. This is the first phytochemical study of the bark of Tectona grandis reforestation specimen in Brazil. The main interest in this work is proving the presence of tectoquinone in reforestation specimen.
\end{abstract}

Keywords: Tectona grandis, Verbenaceae, reforestation specimen, anthraquinones, naphtoquinones.

\section{INTRODUÇÃO}

O plantio de espécies arbóreas em linha nas propriedades rurais, no mundo todo, é considerado como uma modalidade de sistema agroflorestal, devido, basicamente, à possibilidade de haver combinações com cultivos agrícolas e pastagens naturais ou cultivadas (Beer, 1994). A Amazônia, embora ainda possua vastos recursos florestais, quando comparada aos padrões internacionais, sofreu considerável desmatamento. A maior parte da exploração de florestas nativas na Amazônia está sendo conduzida em bases insustentáveis, à medida que grandes áreas de floresta são desmatadas para outros usos da terra, a despeito dos regulamentos que pretendem assegurar o bom manejo de florestas e limitar a conversão da terra para outros usos.

A teca (Tectona grandis L.f.), também conhecida por teak (Índia, Siam, Birmânia e Indonésia), teck (França), ojati (Java), may sak (Laos) e tiek (Alemanha), é uma espécie arbórea da família Verbenaceae que apresenta alto valor comercial. O principal produto desta espécie é a madeira, muito utilizada na carpintaria, na marcenaria, na produção de peças de usos nobres e de móveis finos e, especialmente, na indústria da construção naval, onde é praticamente insubstituível, pelo fato de resistir ao sol, ao calor, ao frio e à água de chuvas e do mar (Rondon Neto et al., 1998). A combinação de beleza, resistência e durabilidade fizeram da madeira desta espécie uma das mais valiosas do mundo, superando outras madeiras nobres, como a do mogno (Swietenia macrophylla King) (Rondon Neto et al., 1998).

Estudos químicos anteriores de teca registraram a presença de uma substância pertencente à classe das antraquinonas, a tectoquinona (1), à qual são atribuídas propriedades antifúngicas, bactericidas e repelentes a ataques de alguns insetos, sendo por isso responsabilizada pela durabilidade da madeira quando exposta aos rigores do tempo (Rudman; Costa, 1958; Ranganathan et al, 1949; Sandermann; Simatupang, 1966). Além da tectoquinona, outras substâncias foram encontradas na espécie Tectona grandis, estas são: 1-hidróxi-2-metilantraquinona, tectol, 5-hidroxilapachol, lapachol, dioxilapachol, desidro- $\alpha$ lapachona, 2-metilquinizarina, pachibasina, $\beta$-sitosterol, obtusifolina, esqualeno, ácido betulínico e até mesmo uma naftoquinona-antraquinona, a tectograndona (Rameshwar; Seshadri, 1979; Singh; Jain; Bhargara, 
1989; Aguinaldo et al., 1993).

Tendo o conhecimento de que a planta produz tectoquinona na idade adulta, o presente estudo fitoquímico foi realizado objetivando a caracterização da presença de tectoquinona (1) em amostras de madeira de espécimes de teca, oriundas de plantios racionais de reflorestamento com idade de, aproximadamente, 10 anos, visto que havia dúvidas se esta substância estava presente ou não, principalmente em amostras de teca não adultas, considerando-se que em regiões aptas da Amazônia para reflorestamento, a expectativa de obter uma rotação econômica de teca é em torno de 10 a 25 anos (Figueredo, 2001).

\section{MATERIAIS E MÉTODOS}

\section{Procedimentos gerais}

Foram realizadas colunas cromatográficas utilizando-se sílica-gel 70-230 Mesh (Merck). A revelação das placas cromatográficas foi feita utilizando-se solução ácida de sulfato cérico e/ou luz UV $\left(\lambda_{\max }=254 \mathrm{~nm}\right)$. Os espectros de RMN foram obtidos em um aparelho VARIAN Mercury $300\left({ }^{1} \mathrm{H}\right.$ a $\left.300 \mathrm{MHz}\right)$.

\section{Material vegetal}

A coleta do material botânico foi feita na área de reflorestamento da empresa Tramontina Belém S.A., situado no município de Aurora do Pará, pelo responsável da plantação, Eng. Florestal Evaristo Terezo, em setembro de 2003. As árvores são originárias de sementes de plantios feitos em Cáceres, no estado de Mato Grosso. Uma exsicata ( $n^{\circ}$ 63348) está depositada no Herbário da EMBRAPA-CPATU.

\section{Extração e isolamento}

O caule de um espécime de teca com 10 anos de idade foi seco em estufa de circulação de ar e moído em um moinho de facas obtendo-se $610 \mathrm{~g}$ de material seco e moído. A partir deste material foi preparado o extrato hexânico através de percolação por 48 horas, a temperatura ambiente. O processo de extração foi repetido duas vezes, sendo que para cada extração foram utilizados 2 litros de hexano, seguido de filtração e evaporação do solvente em um evaporador rotativo a vácuo, obtendo-se $15,1 \mathrm{~g}$ de extrato.

O extrato hexânico foi fracionado por cromatografia em coluna por via úmida (CCVU) filtrante, utilizando-se sílica gel retificada como fase estacionária, submetendo-a aos seguintes sistemas de eluição de 1 litro cada: 1 - hexano 100\%; 2 - hexano/AcOEt 10\%; 3 - hexano/AcOEt 15\%; 4 - hexano/AcOEt 20\%; 5 hexano/AcOEt 30\%; 6 - hexano/AcOEt $50 \%$ e 7 - AcOEt $100 \%$. Após análise espectral de $\mathrm{RMN}$ de ${ }^{1} \mathrm{H}$ foram selecionadas as frações 2 e 3 , pois estas mostraram-se bastante promissoras para serem refracionadas, visto que mostraram sinais referentes a hidrogênios aromáticos. Assim, a fração $2(8,14 \mathrm{~g})$ foi refracionada em coluna de sílica gel nos sistemas, também de 1 litro cada: A - hexano 100\%; B - hexano/AcOEt 2,5\%, deste sistema utilizou-se 2 litros, pois observou-se que o eluído em 1 litro ainda encontrava-se muito concentrado e as frações resultantes foram, então, denominadas de B1 e B2 na ordem de saída do solvente na coluna; $\mathrm{C}$ - hexano/AcOEt 5\%; D - hexano/ AcOEt 10\%; E - hexano/AcOEt 30\% e F - hexano/AcOEt $50 \%$. Após análise espectral de $\mathrm{RMN}{ }^{1} \mathrm{H}$ destas frações foi selecionada a B2 para ser refracionada, pois evidenciava a presença de sinais característicos de hidrogênios de antraquinonas. Este refracionamento rendeu 123 frações, que foram analisadas por cromatografia em camada delgada (CCD), permitindo a reunião das amostras semelhantes, obtendo-se em seguida espectros de RMN ${ }^{1} \mathrm{H}$ das reuniões. Nas frações 36 a 43 foi encontrada a tectoquinona (1) $(27,1 \mathrm{mg})$.

$\mathrm{Na}$ fração B1 foi notado o aparecimento de pequenos cristais alaranjados, os quais foram lavados com hexano e resfriados a aproximadamente $2{ }^{\circ} \mathrm{C}$, desta forma foram obtidos aproximadamente $220 \mathrm{mg}$ destes cristais, e que após análise de seus espectros de $\mathrm{RMN}{ }^{1} \mathrm{H}$ $\mathrm{e}^{13} \mathrm{C}$ ficou caracterizada a presença do lapachol (2).

A fração 3 (hexano/AcOEt 15\%) foi submetida à CCVU, utilizando-se como eluentes misturas de hexano e AcOEt, em sistemas de ordem crescente de polaridade, rendendo 210 frações, as quais foram monitoradas por CCD e reunidas em 25 frações. Na reunião das frações 48 a 65 houve a formação de cristais alaranjados, que também foram lavados com hexano, resfriado a aproximadamente $2{ }^{\circ} \mathrm{C}$, filtrado e evaporado o solvente restante, rendendo 3,7 mg de um sólido, o qual foi analisado por $\mathrm{RMN}{ }^{1} \mathrm{He} \mathrm{e}^{13} \mathrm{C}$, permitindo concluir que tratava-se da obtusifolina (3). A partir das frações 30 a 47, utilizando-se cromatografia em camada preparativa e hexano/AcOEt 10\% como eluente, foi isolada a desidro- $\alpha$-lapachona (4).

\section{RESULTADOS E DISCUSSÃO}

A análise do espectro de $\mathrm{RMN}{ }^{1} \mathrm{H}$ da substância 1 (tabela 1) permitiu caracterizá-la como uma substância aromática, contendo vários hidrogênios que absorvem em região do espectro com valores de $\delta$ mais elevados. Foram observados três sinais típicos de um sistema de acoplamento AMX em $\delta_{\mathrm{H}} 8,11 d(J=1,3 \mathrm{~Hz}), 8,20 d d$ $(J=8,0$ e $1,3 \mathrm{~Hz})$ e $7,60 d(J=8,0 \mathrm{~Hz})$, sendo os dois primeiros atribuídos, respectivamente, aos hidrogênios aromáticos peri à carbonila $\mathrm{H}-1 \mathrm{e} \mathrm{H}-4$ e o terceiro ao hidrogênio H-3 de um esqueleto antraquinônico. Outros dois sinais foram observados nesta região, centrados em $\delta_{\mathrm{H}} 8,31$ e 7,79, cada um integrando dois hidrogênios e atribuídos aos outros quatro hidrogênios da antraquinona. $\mathrm{Na}$ região de alifáticos observa-se apenas um simpleto $(3 \mathrm{H})$, em $\delta_{\mathrm{H}} 2,54$ e atribuído a uma metila ligada a carbono aromático, a qual foi confirmada no espectro de $\mathrm{RMN}{ }^{13} \mathrm{C}$ 
<smiles>Cc1ccc2c(c1)C(=O)c1ccccc1C2=O</smiles><smiles>COc1c(O)c(C)cc2c1C(=O)c1c(O)cccc1C2=O</smiles>

3<smiles>CC(C)=CCC1=C(O)C(=O)c2ccccc2C1=O</smiles>

2<smiles>CC1(C)C=CC2=C(O1)C(=O)c1ccccc1C2=O</smiles>

4

Tabela 1. Dados de $\mathrm{RMN}{ }^{1} \mathrm{H}\left(\mathrm{CDCl}_{3}, 300 \mathrm{MHz}\right)$ de 1, 2, 3 e 4.

\begin{tabular}{|c|c|c|c|c|}
\hline \multicolumn{5}{|c|}{ Compostos } \\
\hline $\mathrm{H}$ & 1 & 2 & $\mathbf{3}$ & 4 \\
\hline & $\delta_{\mathrm{H}} \operatorname{mult}(J \mathrm{~Hz})$ & $\delta_{\mathrm{H}}$ mult $(J \mathrm{~Hz})$ & $\delta_{\mathrm{H}} \operatorname{mult}(\mathrm{J} \mathrm{Hz})$ & $\delta_{\mathrm{H}}$ mult $(J \mathrm{~Hz})$ \\
\hline 1 & $8,11 d(1,3)$ & & & \\
\hline 3 & $7,60 d(8,0)$ & & & \\
\hline 4 & $8,20 d d(8,0$ e 1,3$)$ & & $7,99 d(0,6)$ & \\
\hline 5 & $8,31 \mathrm{~m}$ & $8,1 d d(6,4$ e 1,3$)$ & $7,76 d d(7,5$ e 1,2$)$ & $8,09 d d(6,6$ e 2,7$)$ \\
\hline 6 & $7,79 \mathrm{~m}$ & $7,7 t d(6,4$ e 1,3$)$ & $7,63 d d(8,4$ e 7,5$)$ & $7,80 \mathrm{~m}$ \\
\hline 7 & $7,79 \mathrm{~m}$ & $7,7 t d(6,4$ e 1,3$)$ & $7,26 d d(8,4$ e 1,2$)$ & $7,80 \mathrm{~m}$ \\
\hline 8 & $8,31 \mathrm{~m}$ & $8,1 d d(6,4$ e 1,3$)$ & & $8,09 d d(6,6$ e 2,7$)$ \\
\hline 1 ' & & $3,30 d(6,7)$ & & \\
\hline 2 ' & & $5,20 t(6,7)$ & & \\
\hline 3 , & & & & $5,73 d(10,2)$ \\
\hline $4^{\prime}$ & & $1,65 s$ & & $6,66 d(10,2)$ \\
\hline 5 & & $1,75 s$ & & $1,57 \mathrm{~s}$ \\
\hline 6 & & & & $1,56 s$ \\
\hline $\mathrm{Me}$ & $2,54 s$ & & $2,41 d(0,6)$ & \\
\hline $\mathrm{OMe}$ & & & $4,01 s$ & \\
\hline $\mathrm{OH}-2$ & & $7,20 s$ & $6,83 s$ & \\
\hline $\mathrm{OH}-8$ & & & $12,71 s$ & \\
\hline
\end{tabular}

(tabela 2) pelo sinal em $\delta_{\mathrm{C}} 21,9$. Este espectro também apresentou 10 sinais na região de carbonos aromáticos e ainda mais dois sinais típicos de carbonila em $\delta_{\mathrm{C}} 183,4 \mathrm{e}$ 183,0, caracterizando um núcleo antraquinônico. Todos estes dados foram comparados com os da literatura para a tectoquinona (Itokawa et al., 1991) e mostraram-se superpostos.

Analisando-se o espectro de $\mathrm{RMN}{ }^{1} \mathrm{H}$ de $\mathbf{3}$ (tabela 1), observa-se um sinal simpleto em $\delta_{\mathrm{H}} 12,71$ que é típico de hidroxila aromática quelada à carbonila. O conjunto de três duplos dupletos, todos integrando um hidrogênio, em $\delta_{\mathrm{H}}$ 7,76 $(J=7,5$ e 1,2 Hz), 7,63 $(J=8,4$ e 7,5 Hz) e 7,26 ( $J=8,4$ e 1,2 Hz) é característico de hidrogênios aromáticos com padrões de acoplamento do tipo orto-meta, orto-orto e orto-meta, respectivamente. Em $\delta_{\mathrm{H}} 4,01$ é observado um simpleto, integrando para três hidrogênios, característico de grupo metoxila ligado a anel aromático. O sinal dupleto em $\delta_{\mathrm{H}} 7,99(J=0,6 \mathrm{~Hz}$, $1 \mathrm{H})$, é típico de hidrogênio aromático peri à carbonila. Este hidrogênio é vizinho a um grupo metila, fato que explica o acoplamento a longa distância $(J=0,6 \mathrm{~Hz})$ observada, pela primeira vez para este composto, tanto no sinal do hidrogênio aromático como no sinal relativo ao grupo metila ligada ao anel em $\delta_{\mathrm{H}} 2,41(d ; J=0,6 \mathrm{~Hz}$, 
Tabela 2. Dados de $\mathrm{RMN}^{13} \mathrm{C}\left(\mathrm{CDCl}_{3}, 300 \mathrm{MHz}\right)$ de $\mathbf{1}, 2$ e 3.

\begin{tabular}{|c|c|c|c|}
\hline \multicolumn{4}{|c|}{ Compostos } \\
\hline${ }^{13} \mathrm{C}$ & 1 & 2 & 3 \\
\hline & $\delta_{\mathrm{C}}$ & $\delta_{\mathrm{C}}$ & $\delta_{\mathrm{C}}$ \\
\hline 1 & 127,4 & 184,5 & 146,3 \\
\hline 2 & 145,2 & 152,7 & 154,2 \\
\hline 3 & 134,9 & 123,5 & 131,3 \\
\hline 4 & 127,5 & 181,7 & 126,4 \\
\hline 5 & 127,1 & 126,0 & 119,1 \\
\hline 6 & 133,9 & 134,8 & 136,3 \\
\hline 7 & 134,0 & 132,8 & 123,7 \\
\hline 8 & 127,1 & 126,7 & 162,1 \\
\hline 9 & 183,4 & & 187,9 \\
\hline 10 & 183,0 & & 181,8 \\
\hline $4 a$ & 131,3 & 132,9 & 125,9 \\
\hline $8 \mathrm{a}$ & 133,6 & 129,4 & 115,8 \\
\hline $9 \mathrm{a}$ & 133,4 & & 123,9 \\
\hline $10 \mathrm{a}$ & 133,6 & & 134,5 \\
\hline $\mathrm{Me}$ & 21,9 & & 16,3 \\
\hline 1 ' & & 22,6 & \\
\hline 2 ' & & 119,6 & \\
\hline $3^{\prime}$ & & 133,8 & \\
\hline $4^{\prime}$ & & 17,8 & \\
\hline 5 & & 25,7 & \\
\hline $\mathrm{OMe}$ & & & 62,2 \\
\hline
\end{tabular}

3H). $\mathrm{O}$ espectro de $\mathrm{RMN}{ }^{13} \mathrm{C}$ de 3 (tabela 2) apresenta dois sinais relativos às carbonilas $\mathrm{C}-9$ e $\mathrm{C}-10$ em $\delta_{\mathrm{C}} 187,9$ e 181,8 , respectivamente, sendo esta grande diferença de deslocamento atribuída à quelação de apenas uma das carbonilas com o grupo hidroxila. Outros sinais de carbono importantes observados foram aqueles em $\delta_{\mathrm{C}}$ 62,2, que caracteriza uma metoxila di-ortosubstituída na posição 1 , e em $\delta_{\mathrm{C}} 16,3$, que comprova a presença de grupo metila na posição 3 . Todos esses dados foram coincidentes com os encontrados na literatura para a obtusifolina (Takido, 1958).

No espectro de $\mathrm{RMN}{ }^{1} \mathrm{H}$ de 2 (tabela 1), são observados sinais característicos de grupo prenila em $\delta_{\mathrm{H}}$ $3,30 d(6,7) ; 5,20 t(6,7) ; 1,65$ e 1,75 ( $s, 3 \mathrm{H}$ cada), bem como sinais de hidrogênios aromáticos característicos da naftoquinona lapachol, que são dois tripletos de dupletos em $\delta_{\mathrm{H}} 7,7$ referentes a H-6 e H-7 e dois duplos dupletos em $\delta_{\mathrm{H}} 8,1$ referentes a $\mathrm{H}-5$ e $\mathrm{H}-8$, além do sinal de hidroxila ligada a carbono $\mathrm{sp}^{2}$ em $\delta 7,2$. Assim como os dados do espectro de RMN ${ }^{1} \mathrm{H}$ de 2 (Khan et al., 1999), também os dados espectrais de $\mathrm{RMN}{ }^{13} \mathrm{C}$ (tabela 2), foram compatíveis com aqueles encontrados na literatura para o lapachol(Viana, 1999), principalmente os sinais atribuídos aos dois grupos carbonila em $\delta_{\mathrm{C}} 184,5$ e 181,7 e aqueles relacionados aos carbonos do grupo $\mathrm{CH}_{2} \mathrm{CH}=\mathrm{C}\left(\mathrm{CH}_{3}\right)_{2}$, respectivamente em $\delta_{\mathrm{C}} 22,6,119,6,133,8,17,8$ e 25,7.

No espectro de $\mathrm{RMN}{ }^{1} \mathrm{H}$ de 4 (tabela 1) são observados dois sinais de hidrogênios aromáticos: um duplo dupleto em $\delta_{H} 8,09(J=6,6$ e $2,7 \mathrm{~Hz}, 2 \mathrm{H})$ e um multipleto em $\delta_{\mathrm{H}} 7,80(2 \mathrm{H})$, além dos sinais que indicam a presença de um anel pirano em $\delta_{\mathrm{H}} 6,66(d, J=10,2 \mathrm{~Hz})$; $5,73(d, J=10,2 \mathrm{~Hz}) ; 1,57(s)$ e $1,56(s)$. Estes dados estão coerentes com os dados de $\mathrm{RMN}{ }^{1} \mathrm{H}$ da desidro- $\alpha$ lapachona (Weinberg et al, 1970).

\section{CONCLUSÃO}

Os resultados obtidos neste trabalho, com ênfase para o isolamento da tectoquinona, foram importantes, pois atestam que espécimes de reflorestamento de teca com idade de 10 anos são capazes de biossintetizar a antraquinona que é responsabilizada por garantir a durabilidade da madeira obtida de teca.

Além da tectoquinona, outras três substâncias foram isoladas neste trabalho, a obtusifolina, a desidro$\alpha$-lapachona e o lapachol, as quais também foram obtidas em trabalhos fitoquímicos anteriores de teca, sendo que o lapachol é uma substância muito comum na família Verbenaceae e que possui potente atividade antibacteriana (Gafner et al., 1996; Cortes et al., 1983), podendo também estar relacionado com a durabilidade da madeira. Esta idéia é reforçada pelo relato da presença desta substância em algumas espécies de ipê (Tabebuia serratifolia G. Don., Tabebuia heptaphylla (vell) Mart., Tabebuia chryssotricha Mart., Tabebuia ipe Mart.) (Ferreira, 1996), que também produzem madeiras resistentes aos rigores do clima e de agentes biológicos.

\section{AGRADECIMENTOS}


Os autores agradecem ao $\mathrm{CNPq}$ pelo suporte financeiro, ao Eng. Evaristo Terezo pelo fornecimento do material botânico e a UFPA pelo espaço cedido para a pesquisa realizada.

\section{REFERÊCIAS}

Aguinaldo AM, Ocampo OPM, Bowden BF, Gray AI, Waterman PG 1993. Tectograndone, an anthraquinonenaphthoquinone pigment from the leaves of Tectona grandis. Phytochemistry 33: 933-935.

Beer FJ 1994. Consideraciones basicas para el establecimiento de especies maderables en linderos. Agroforesteria en las Américas 1: 21-24.

Cortes M, Katalinic J, Valderrama J 1983. Studies on quinones $\mathrm{X}$. Heterocyclic derivatives of lapachol as potential antimicrobial agents. An Quim 79: 202.

Ferreira VF 1996. Aprendendo sobre os conceitos de ácidos e bases. Química Nova na Escola 4: 35-36.

Figueredo EO 2001. Reflorestamento com teca (Tectona grandis L.f.) no estado do Acre. Rio Branco: Embrapa Acre. 28p. (Embrapa Acre. Documento 65).

Gafner S, Wolfender JL, Nianga M, Stoeckli-Evans H, Hostettmann K 1996. Antifungal and antibacterial naphthoquinones from Newbouldia laevis roots. Phytochemistry 42: 1315-1320.

Itokawa I, Qiao Y, Takeya K 1991. Anthraquinones, naphthoquinones and naphthohydroquinones from Rubia oncotrichia. Phytochemistry 30: 637-640.

Khan RM,Mlungwana SM 1999.5-Hydroxylapachol: acytotoxic agent from Tectona grandis. Phytochemistry 50: 439-442.

Rameshwar J, Seshadri TR 1979. Chemical investigation of Tectona grandis (roots). J. Indian Chem. Soc. 56: 940-941.

Ranganathan SK, Koshi T, Sitaraman NL 1949. Methylanthraquinone (Tectoquinone), a synergist for 2,2-bis(p-chlorophenyl)-1,1,1-trichloroethane (DDT). Nature 164 (4182): 1095.

Rondon Neto RM, Macedo RLG, Tsukamoto Filho AA 1998. Formação de povoamentos florestais com Tectona grandis L.f. (Teca). Boletim Técnico - Série Extensão 7 (33): 1-29.

Rudman P, Da Costa EWB 1958. Relationship of tectoquinone to durability in Tectona grandis. Nature 181: 721722.

Sandermann W, Simatupang MH 1966. Zur chemie und biochemie des Teakholzes (Tectona grandis L.). Holz Roh-Werkst 24: 190.

Singh P, Jain S, Bhargara S 1989. A 1,4-anthraquinone derivative from Tectona grandis. Phytochemistry 28: 12581259.

Takido M 1958. Constituents of the seeds of Cassia obtusifolia. I. The structure of obtusifolin. Chem Pharm Bull 6: 7397-7400.

Viana EP 1999. Estudo Fitoquímico e Farmacológico de Bauhinia guianensis Aubi. Belém, 88p. Dissertação de Mestrado - Programa de Pós-Graduação em Química, Universidade Federal do Pará.

WeinbergMLD, GottliebOR, Oliveira GG 1970. Naphtoquinones from Zeyhera tuberculosa. Phytochemistry 15: 570 .

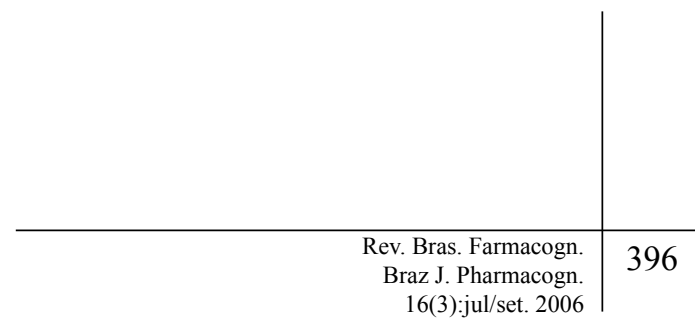

